\title{
Certeza diagnóstica del SOFA-simplificado en pacientes con COVID-19 en Unidad de Terapia Intensiva del Centro Médico ABC
}

Diagnostic certainty of the SOFA-simplified in patients with COVID-19

in the Intensive Therapy Unit of the ABC Medical Center

Certeza diagnóstica do SOFA simplificado em pacientes com COVID-19 na

Unidade de Terapia Intensiva do Centro Médico ABC

\author{
María Guadalupe Gómez García, ${ }^{*}$ Enrique Monares Zepeda, ${ }^{\star}$ Juvenal Franco-Granillo, ${ }^{*}$ Janet Silvia Aguirre-Sánchez, \\ Rodrigo Chaires Gutiérrez, ${ }^{*}$ María Vigil Escala Bejarano*
}

\section{RESUMEN}

Introducción: La pandemia de la enfermedad por coronavirus 2019 (COVID-19) continúa desafiando los sistemas de atención médica en todo el mundo. En los últimos dos meses, sólo unos pocos estudios han analizado los factores pronósticos de muerte en pacientes con COVID-19. Por esto, es de gran relevancia clínica que existan análisis de escalas pronósticas que ayuden a anteponerse a la gravedad de pacientes para la toma de decisiones y acciones oportunas que eviten complicaciones y aumento en insumos hospitalarios. En el presente estudio se propone la utilización de la escala SOFA-simplificado, que es una escala pronóstica que consiste en la evaluación clínica, sin requerimientos de estudios de gabinete.

Objetivos: Demostrar la certeza diagnóstica mediante la concordancia y el grado de ésta al utilizar SOFA-simplificado y SOFA.

Material y métodos: Estudio de cohorte retrospectivo en pacientes con COVID-19 que ingresaron a la UTI. Se recolectaron datos demográficos, resultados de laboratorio e indicadores inmunológicos, con la finalidad de analizar y comparar las escalas pronósticas. Las variables cualitativas se reportan como frecuencias y proporciones, las cuantitativas como mediana con rango intercuartil o media con desviación estándar. Se calcula el tamaño de muestra con Epi Info versión 5.4.6 y OpenEpi. Se comparan ambas escalas pronósticas, así como supervivencia de Kaplan Meier. Se analiza la superposición de ambas escalas para demostrar la certeza diagnóstica mediante curvas de ROC, se valora el área bajo la curva y para la comparación entre ellas se realizará agreement con coeficiente de correlación intraclase $(\mathrm{CCl})$ y representación por Bland-Altman. El programa que se utilizó para análisis de datos fue IBM SPSS Statistics 24, aplicación Epi Info versión 5.4.6 y OpenEpi.

Resultados: El grado de concordancia medido por índice de correlación intraclase fue de 0.688 (IC 95\% 0.405-0.836) con p significativa $(p<0.001)$, se traduce en un alto grado de concordancia entre SOFA y SOFA simplificado y un rendimiento similar. Conclusión: Según el grado de concordancia tan elevado que presenta en este estudio, se podría utilizar la escala SOFA simplificada como sustitución para predecir gravedad en pacientes con COVID en áreas de salud en las que no se cuente con equipo de laboratorio, identificando oportunamente gravedad, mejorar la atención y disminuir mortalidad.

Palabras clave: SOFA, SOFA-simplificado, índice de correlación intraclase.

\section{ABSTRACT}

Introduction: The 2019 coronavirus disease (COVID-19) pandemic continues to challenge health care systems around the world. In the past two months, only a few studies have looked at prognostic factors for death in COVID-19 patients. It is therefore of great clinical relevance that there are analysis of prognostic scales, which help to prevail over the severity of patients, for decision-making and timely actions that avoid complications and increase in hospital supplies. In the present study, the use of the simplified SOFA scale is proposed, which is a prognostic scale that consists of clinical evaluation, without any requirements for office studies. Objectives: To demonstrate the diagnostic certainty through the concordance and degree of agreement of using SOFA-simplified and SOFA.

Material and methods: Retrospective cohort study in COVID-19 patients admitted to ICU. Demographic data, laboratory results and immunological indicators were collected, in order to analyze and compare prognostic scales. Qualitative variables are reported as frequencies and proportions. Quantitative variables such as

\section{* Centro Médico ABC. Ciudad de México, México.}

Recibido: 16/10/2020. Aceptado: 27/10/2020.

Citar como: Gómez GMG, Monares ZE, Franco-Granillo J, Aguirre-Sánchez JS, Chaires GR, Escala BMV. Certeza diagnóstica del SOFA-simplificado en pacientes con COVID-19 en Unidad de Terapia Intensiva del Centro Médico ABC. Med Crit. 2021;35(1):18-22. https://dx.doi.org/10.35366/99149 median with interquartile range or mean with standard deviation. The sample size is calculated with Epi Info version 5.4.6 and OpenEpi. Both prognostic scales are compared; as well as, survival of Kaplan Meier. The superposition of both scales is analyzed to demonstrate the diagnostic certainty by means of ROC curves, the area under the curve is assessed and for the comparison between them an agreement with intraclass correlation coefficient (ICC) and representation by Bland Altman will be made. The program to use for data analysis IBM SPSS Statistics 24, application Epi Info version 5.4.6 and OpenEpi.

Results: The degree of agreement measured by intraclass correlation index was $0.688(95 \% \mathrm{Cl} 0.405-0.836)$ with significant $p(p<0.001)$, which translates into a high degree of agreement between SOFA and simplified SOFA and a similar performance. Conclusion: According to the high degree of agreement presented in this study, the simplified SOFA scale could be used as a substitute to predict severity in patients with COVID in health areas where laboratory equipment is not available, identifying the severity in a timely manner, improve timely care and reduce mortality. Keywords: SOFA, SOFA-simplified, intraclass correlation index.

\section{RESUMO}

Introdução: A pandemia da doença coronavírus de 2019 (COVID-19) continua a desafiar os sistemas de saúde em todo o mundo. Nos últimos 2 meses, apenas alguns estudos avaliaram fatores prognósticos para morte em pacientes com COVID-19. Por esse motivo, é de grande relevância clínica que existam análises de escalas de prognóstico, que auxiliem a prevalecer sobre a gravidade dos pacientes, para a tomada de decisões e ações oportunas que evitem complicações e aumento de materiais hospitalares. No presente estudo, é proposta a utilização da escala SOFA simplificada, que é uma escala de prognóstico que consiste na avaliação clínica, sem necessidade de estudos de consultório.

Objetivos: Demonstrar a certeza diagnóstica pela concordância e grau do uso do SOFA simplificado e do SOFA.

Material e métodos: Estudo de coorte retrospectivo em pacientes COVID-19 internados em UTI. Dados demográficos, resultados laboratoriais e indicadores imunológicos foram coletados para análise e comparação das escalas de prognóstico. As variáveis qualitativas são relatadas como frequências e proporções. Variáveis quantitativas como mediana com intervalo interquartil ou média com desvio padrão. O tamanho da amostra é calculado com Epi Info versão 5.4.6 e OpenEpi. Ambas escalas de prognóstico são comparadas; bem como a sobrevivência de Kaplan Meier. A sobreposição das duas escalas é analisada para demonstrar a certeza diagnóstica por meio de curvas ROC, a área sob a curva é avaliada e para a comparação entre elas será feita uma concordância com o coeficiente de correlação intraclasse (ICC) e representação de Bland Altman. O programa a ser usado para análise de dados IMB SPSS STATISTICS 24, aplicativo Epi Info versão 5.4 .6 e OpenEpi.

Resultados: O grau de concordância medido pelo índice de correlação intraclasse foi de 0.688 (IC95\% 0.405-0.836) com $p$ significativo $(p<0.001)$, o que se traduz em alto grau de concordância entre SOFA e SOFA simplificado e desempenho semelhante.

Conclusão: De acordo com o alto grau de concordância apresentado neste estudo, a escala SOFA simplificada poderia ser utilizada como substituto para predizer a gravidade em pacientes com COVID em áreas da saúde onde não há equipamentos laboratoriais disponíveis, identificando a gravidade em tempo hábil, melhorar o atendimento oportuno e reduzir a mortalidade.

Palavras-chave: SOFA, SOFA simplificado, índice de correlação intraclasse.

\section{INTRODUCCIÓN}

A principios de diciembre de 2019 , se produjo un brote de la enfermedad por coronavirus 2019 (COVID-19), 
causado por un nuevo síndrome respiratorio agudo severo coronavirus 2 (SARS-CoV-2), ocurrió en la ciudad de Wuhan, provincia de Hubei, China. El 30 de enero de 2020, la Organización Mundial de la Salud declaró el brote como una emergencia de salud pública de preocupación internacional. ${ }^{1}$

Un nuevo coronavirus fue identificado como el agente causante y posteriormente fue denominado COVID-19 por la Organización Mundial de la Salud (OMS). Considerado un pariente del síndrome respiratorio agudo severo (SARS) y el síndrome respiratorio de Oriente Medio (MERS), COVID-19 es causado por un betacoronavirus llamado SARS-CoV-2 que afecta el tracto respiratorio inferior y se manifiesta como neumonía en humanos. ${ }^{2}$

EI SARS-CoV-2 es un virus binuclear que tiene un amplio espectro clínico de infección. En la infección pulmonar por SARS-CoV se observa daño alveolar difuso, proliferación de células epiteliales y un aumento de macrófagos. La linfopenia, la hemofagocitosis en el pulmón, además de la atrofia de la pulpa blanca del bazo observada en pacientes con SARS son similares a las infecciones fatales por el virus de la influenza H5N1.

En los seres humanos, las infecciones por SARSCoV afectan principalmente al tracto respiratorio superior y al tracto gastrointestinal y varían desde una enfermedad leve y autolimitada, como el resfriado común, hasta manifestaciones más graves, como bronquitis y neumonía con afectación renal. ${ }^{3}$

En los últimos dos meses, sólo unos pocos estudios han analizado los factores pronósticos de muerte en pacientes con COVID-19, con énfasis repetido en factores como la edad, linfocitosis, leucocitosis y ALT elevada. Es por ello que surge la necesidad de conocer variables que nos orienten a anteponernos a la gravedad; sin embargo, en la mayoría de escalas pronósticas se requiere de estudios de laboratorio o gabinete, con los que en muchas ocasiones no se cuenta. En el presente estudio se propone la utilización de la escala simplificada SOFA (Sequential Organ Failure Assessment Score), sin requerimientos de estudios, más allá que la clínica. Se realiza este estudio con la finalidad de demostrar que existe correlación entre ambas escalas en cuanto a sensibilidad y especificidad.

Se plantea la utilización de una escala simplificada, minimizando costos de laboratorios o para aquellas instituciones de primer nivel que no es posible obtener laboratorio, por ello es difícil estadificar en una escala pronóstica al paciente, minimizando la gravedad y retrasando así la atención oportuna, aumentando la mortalidad.

Una forma de maximizar la eficacia de un equipo es el empleo de una lista de cotejo, que forme parte de una escala pronóstico, para evitar omitir datos que nos orienten en la gravedad y poder actuar de manera oportuna. ${ }^{4}$

Existen múltiples escalas que nos permiten homogenizar criterios, pero muchas veces las escalas consumen tiempo o, en su mayoría, requieren recursos con los que no se cuenta en todos las instituciones. Es por ello que se sugiere el empleo de las modificaciones de la escala de SOFA (Sa-SOFA) para un rápido pase de visita, determinar la gravedad del paciente y el foco de deterioro. Una escala de Sa-SOFA en aumento debe alertarnos de que la evolución del paciente no es la adecuada e identifica el órgano al que debemos prestar atención (Tabla 1). ${ }^{5}$

RESPUESTA MOTOR: uno de los componentes de mayor valor predictivo pronóstico de la escala de SOFA proviene de su componente neurológico. Sin mencionar que la preservación del cerebro es una de nuestras principales metas aún más allá de la supervivencia. ${ }^{6}$

Por ello, si encontramos un puntaje elevado lo primero que debemos hacer es revalorar sin efectos farmacológicos. Este apartado neurológico se obtiene de la información del componente motor del paciente, ${ }^{7}$ asegurando que el sujeto se encuentre con una sedación optimizada, valorada por escala de agitación y sedación Richmond simplificada (Sa-RASS), y lograr mantener al paciente sin dolor con una analgesia adecuada valorada con Sa-CPOT (escala de dolor y analgesia simplificada). ${ }^{8}$

$\mathrm{SAO}_{2} / \mathrm{FiO}_{2}(\mathrm{~S} / \mathrm{F})$ : aumentos de puntaje en SOFA en el parámetro respiratorio deben hacernos revisar la necesidad de que el caso requiera mayor apoyo respiratorio y encontrar la causa del deterioro.

VASOPRESORES: valorar el comportamiento, si se encuentran en aumento los requerimientos de vasopresores, se debe realizar un protocolo de descarte de sepsis (tomando en cuenta los accesos venosos), si por el contrario existe diminución de éstos, se deben suspender si la estabilidad hemodinámica del paciente lo tolera, así como valorar desescalonamiento de antibioticoterapia. $^{9}$

DIURESIS: vigilar la función renal en pacientes críticos es vital, valorar ajuste de nefrotóxicos (como una de las principal causa de afección renal en pacientes críticos). Ajustar soluciones intravenosas, valorar si es necesario el uso de diurético o apoyo de sustitución renal. ${ }^{10}$

ESCALA DE FALLA GASTROINTESTINAL (GIF): la importancia pronóstica de la nutrición y mirar hacia el futuro. Uno de cada cinco pacientes que no cumplen el

Tabla 1: Escala SOFA simplificado.

\begin{tabular}{lccc}
\hline Puntaje/Criterio & 0 & 1 & 2 \\
\hline Respuesta & 6 & $5 ; 4$ & $\begin{array}{c}<4 \text {, pupilas } \\
\text { anormales }\end{array}$ \\
motora/ocular (RASS) & $>190$ & $190-80$ & $<80$ \\
$\mathrm{SaO}_{2} / \mathrm{FiO}_{2}$ & $<0.75$ & $0.75-2$ & $>2$ \\
Vasopresores $(\mu \mathrm{g} / \mathrm{km} / \mathrm{min})$ & $>2,000$ & $2,000-500$ & $<500$ \\
Diuresis $(\mathrm{mL})$ & $\mathrm{No} / \mathrm{GIF}<2$ & $\mathrm{GIF}>2$ & $\mathrm{Sí}$ \\
Ictericia & & & \\
conjuntiva/GIF & & & \\
\hline
\end{tabular}

RASS = al estado neurológico: respuesta motora y ocular. Escala de SOFA-simplificado de Vacheron $\mathrm{CH}$, et al. ${ }^{5}$ 


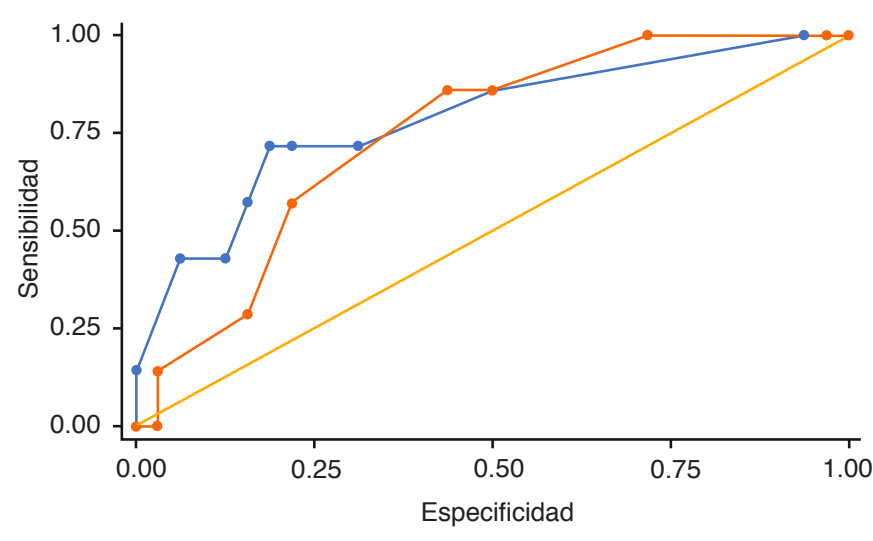

$\longrightarrow$ SOFA área ROC: 0.7857
$\longrightarrow$ SOFA-simplificado área ROC: 0.7478
Referencia

Figura 1: Curva ROC SOFA-simplificado y SOFA.

$80 \%$ de sus requerimientos enterales en los tres primeros días de estancia en terapia intensiva fallecerán en ese internamiento y los sobrevivientes que no pudieron cumplir con sus requerimientos nutricionales identifica a un grupo con alto riesgo de desarrollar secuelas importantes con altos costos humanos y materiales en las etapas de rehabilitación del síndrome de PICS. Por ello, la insistencia del aporte nutricional enteral. Por último, la única forma de detectar de manera temprana el síndrome compartimental abdominal es no dejar pasar por alto la combinación de un paciente intolerante a la nutrición enteral que también sobrelleva una mala evolución en general.

Es importante determinar las metas de la nutrición enteral y la búsqueda de obstáculos para cumplir estas metas que muchas veces son problemas más grandes que se evidencian después, cuando es más difícil su abordaje.

\section{MATERIAL Y MÉTODOS}

Diseño del estudio: se realizó la selección de pacientes con COVID-19 admitidos en Terapia Intensiva del Centro Médico ABC de la Ciudad de México, desde marzo 2020 a mayo 2020.

Se incluyeron pacientes con las siguientes características: 1) mayores de 18 años; 2) con diagnóstico de COVID-19 mediante RT-PCR multiplex en tiempo real; 3) con registros clínicos completos de historial médico y resultados de laboratorio en expediente TIMSA. Se excluyeron los siguientes casos: 1) sin registro clínico completo en expediente médico del sistema TIMNSA; 2) sin registro de laboratorios en expediente médico TIMNSA. Para el análisis de variables de estudio, se recolectaron datos demográficos, resultados de laboratorio e indicadores inmunológicos, con la finalidad de analizar y comparar escalas pronóstico y laboratorios. Para cada paciente se examinaron los caracteres: SOFA, SOFA simplificado, IL6, PCR, leucocitos, linfocitos, $L D H$, dímero $D$, fibrinógeno, BNP, ferritina, relación plaquetas-linfocitos (RPL), relación neutrófiloslinfocitos. Con la finalidad de medir la concordancia de sensibilidad y especificidad de las escalas SOFA y SOFA-simplificado.

Recolección de los datos: se analizaron expedientes clínicos de pacientes que ingresaron a terapia intensiva con COVID-19 durante los meses de marzo 2020 a mayo 2020, se recabaron las variables para obtener escalas pronósticas como SOFA y SOFA simplificado al ingreso del paciente en terapia intensiva, con la finalidad de comparar sensibilidad y especificidad. Se analizaron también variables que componen SOFA como estado neurológico, vasopresores, creatinina, uresis, bilirrubinas, plaquetas, $\mathrm{PaO}_{2} / \mathrm{FiO}_{2}$, TAM; para el SOFAsimplificado se agrega estado nutricio.

EI programa a utilizar para análisis de datos IBM SPSS Statistics 24, aplicación Epi Info versión 5.4.6 y OpenEpi.

Definición de desenlaces: el desenlace primario en este trabajo es determinar la concordancia entre ambas escalas pronósticas, los desenlaces secundarios, valorar la relación que existe entre escalas pronósticas SOFA y SOFA simplificado, y marcadores infamatorios, como gravedad pronóstica.

Análisis estadístico: las variables cualitativas se reportan como frecuencias y proporciones. Las variables cuantitativas se reportan como mediana con rango intercuartil o media con desviación estándar, dependiendo la normalidad de su distribución. Se calcula el tamaño de muestra con Epi Info versión 5.4.6 y OpenEpi.

Para la evaluación, se utilizaron ambas escalas pronósticas comparando sensibilidad y especificidad entre SOFA y SOFA-simplificado, capacidad predictiva me-

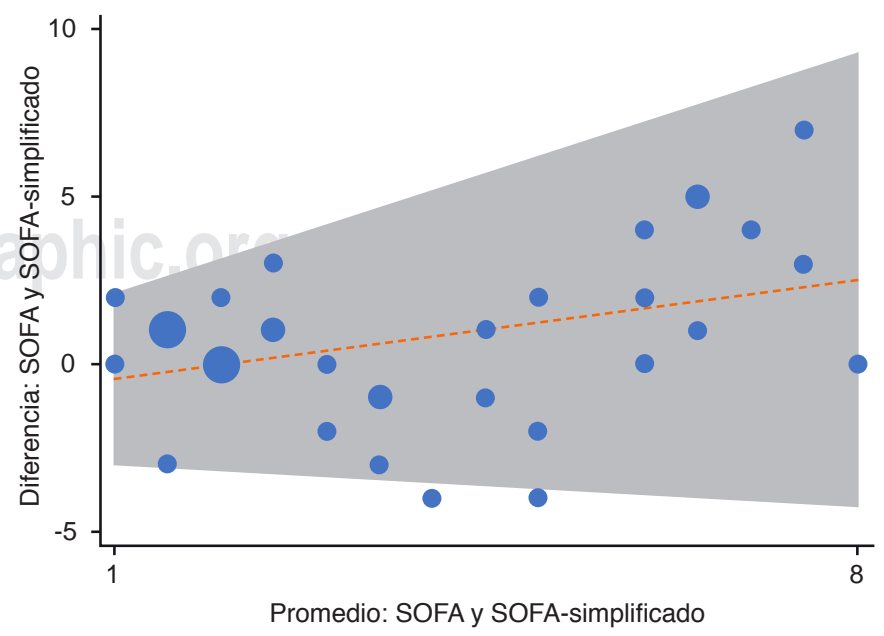

Figura 2: Gráfica de Bland Altman SOFA-simplificado y SOFA. 
Tabla 2: Coeficiente de correlación intraclase.

\begin{tabular}{|c|c|c|c|c|c|c|c|}
\hline & Correlación intraclase* & \multicolumn{2}{|c|}{ Intervalo de confianza 95\% } & \multicolumn{4}{|c|}{ Prueba F con valor verdadero 0} \\
\hline Medidas individuales & $0.524^{\star *}$ & 0.254 & 0.719 & 3.206 & 38 & 38 & 0.000 \\
\hline
\end{tabular}

Modelo de efectos mixtos de dos factores en el que los efectos de las personas son aleatorios y los efectos de las medidas son fijos.

* Coeficientes de correlación intraclase de tipo C utilizando una definición de coherencia, la varianza inter-medidas se excluye de la varianza del denominador.

** El estimador es el mismo, ya esté presente o no el efecto de interacción.

*** Esta estimación se calcula asumiendo que no está presente el efecto de interacción, ya que de otra manera no es estimable.

diante puntos de corte óptimos con curvas de supervivencia de Kaplan Meier.

Después se analizará la superposición de ambas escalas para demostrar la certeza diagnóstica, se realizará primero el análisis de las dos categorías distintas para cada SOFA, se realizarán dos curvas de ROC, se valora el área bajo la curva, y para la comparación entre ellas se realizará agreement con coeficiente de correlación intraclase $(\mathrm{CCl})$ y representación por BlandAltman. Correlaciones bivariadas entre SOFA simplificado y SOFA.

El programa a utilizar para análisis de datos IBM SPSS Statistics 24, aplicación Epi Info versión 5.4.6 y OpenEpi.

\section{RESULTADOS}

Según las gráficas de Bland-Altman, se evidencian entre ambas escalas similitudes en el comportamiento del SOFA, entre más alto es el SOFA que presenten los pacientes, la diferencia entre ambas escalas es menor. Se realizó correlación de Pearson en la cual se evidencia alta correlación entre ambas escalas.

El grado de concordancia medido por índice de correlación intraclase fue de 0.688 (IC 95\% 0.405-0.836) con una $p$ significativa $<0.001$, se traduce en un alto grado de concordancia entre SOFA y SOFA simplificado y un rendimiento similar (Figuras 1 y 2). Con esto se evidencia la concordancia significativa entre ambas escalas, ya que comparando todos los puntajes de SOFA contra todos los otros puntajes de SOFA-simplificado muestran un alto grado de concordancia (Tabla 2). Así como un rendimiento muy similar por el área bajo la curva.

\section{DISCUSIÓN}

Con la actual pandemia, y ante la falta de recursos en algunas instituciones, es imposible realizar laboratorios, con esto se dificulta la clasificación de los pacientes en alguna escala pronóstica y poder identificar con tiempo casos de riesgo a complicarse para actuar en tiempo y forma. En el presente estudio se buscó comparar ambas escalas con la finalidad de poder ser sustituidas en lugares donde no se tuviera disposición de laboratorios.

Se realizó el índice de correlación intraclase $(\mathrm{CCl})$, el cual nos explica la superposición que existe entre ambas escalas pronósticas, un índice cercano a 1 nos habla de mayor concordancia, en el presente estudio se obtiene 0.688 con una p significativa, por lo que se evidencia que existe un alto grado de concordancia entre ambas escalas pronósticas, que se traduce en significancia estadística para la sustitución de ambas.

\section{CONCLUSIONES}

Según el grado de concordancia tan elevado que se presenta en este estudio, se podría utilizar la escala SOFA simplificada como sustitución para predecir gravedad en pacientes con COVID en áreas de salud en las que no se cuente con equipo de laboratorio, identificando oportunamente identificar gravedad, mejorar la atención oportuna y disminuir mortalidad. Esta investigación mostró una concordancia significativa entre ambas escalas, ofreciendo una posibilidad de estadificar oportunamente.

Se necesitan más estudios complementarios y análisis de todas las variables para concluir si estas escalas presentan rendimiento igual entre ambas en cuanto a la mortalidad.

\section{REFERENCIAS}

1. Zhu N, Zhang D, Wang W, Li X, Yang B, Song J, et al. A Novel Coronavirus from patients with pneumonia in China, 2019. N Engl J Med. 2020;382(8):727-733.

2. Informe de agrupación de neumonía de etiología desconocida en la ciudad de Wuhan. Comisión Municipal de Salud de Wuhan, 2019. Disponible en: https://www.ecdc.europa.eu/sites/default/ files/documents/Risk\%20assessment\%20-\%20pneumonia\%20 Wuhan\%20China\%2017\%20Jan\%202020.pdf

3. Serrano-Castro PJ, Estivill-Torrús G, Cabezudo-García P, ReyesBueno JA, Ciano PN, Aguilar-Castillo MJ, et al. Influencia de la infección SARS-CoV-2 sobre enfermedades neurodegenerativas y neuropsiquiátricas: ¿una pandemia demorada? Neurología. 2020;35(4):245-251. Disponible en: https://www.ncbi.nlm.nih. gov/pmc/articles/PMC7164900/\#

4. Aziz S, Arabi YM, Alhazzani W, Evans L, Citerio G, Fischkoff $\mathrm{K}$, et al. Managing ICU surge during the COVID-19 crisis: rapid guidelines. Intensive Care Med. 2020;46(7):1303-1325. 
5. Vacheron $\mathrm{CH}$, Friggeri $\mathrm{A}$, Iwaz J, Allaouchiche $\mathrm{B}$, Bohe J, Monneret G, et al. A new simplified and accurate sa-SOFA score. $J$ Crit Care. 2020;57:240-245. doi: 10.1016/j.jcrc.2019.11.009.

6. Knox DB, Lanspa MJ, Pratt CM, Kuttler KG, Jones JP, Brown SM. Glasgow Coma Scale score dominates the association between admission Sequential Organ Failure Assessment score and 30-day mortality in a mixed intensive care unit population. $J$ Crit Care. 2014;29(5):780-785. doi: 10.1016/j.jcrc.2014.05.009

7. Healey C, Osler TM, Rogers FB, Healey MA, Glance LG, Kilgo PD, et al. Improving the Glasgow Coma Scale score: motor score alone is a better predictor. J Trauma. 2003;54(4):671-578; discussion 678-80. doi: 10.1097/01.TA.0000058130.30490.5D.

8. Telles GP, Ferreira IBB, Carvalho de Menezes R, do Carmo TA, David Pugas PL, Marback LF, et al. Comparison of a modified Sequential Organ Failure Assessment Score using RASS and FOUR. PLoS One. 2020;15(2):e0229199. doi: 10.1371/journal. pone.0229199.

9. Roberts RJ, Miano TA, Hammond DA, Patel GP, Chen JT, Phillips $\mathrm{KM}$, et al. Evaluation of vasopressor exposure and mortality in patients with septic shock. Crit Care Med. 2020;48(10):14451453. doi: 10.1097/CCM.0000000000004476.

10. Avila MO, Zanetta DM, Abdulkader RC, Yu L, Burdmann EA. Urine volume in acute kidney injury: how much is enough? Ren Fail. 2009;31(10):884-890. doi: 10.3109/08860220903216089.

\section{Patrocinios: Ninguno.}

Relación de conflicto de intereses: Ninguno.

\author{
Correspondencia: \\ María Guadalupe Gómez García \\ Artificios Núm. 64 \\ Col. Las Américas, 01120, \\ Alcaldía Álvaro Obregón, \\ Ciudad de México, México. \\ Tel: 68 6946-5709 / 68 6566-5944 \\ E-mail: lupitagomez@gmail.com
}

